\title{
Co-expression of CD133, CD44v6 and human tissue factor is associated with metastasis and poor prognosis in pancreatic carcinoma
}

\author{
KAI CHEN ${ }^{*}$, ZHONGHU LI* ${ }^{*}$ PENG JIANG, XI ZHANG, YUJUN ZHANG, \\ YAN JIANG, YU HE and XIAOWU LI \\ Department of Hepatobiliary Surgery Institute, Southwest Hospital, \\ Third Military Medical University, Chongqing 400038, P.R. China
}

Received March 14, 2014; Accepted May 2, 2014

DOI: 10.3892/or.2014.3245

\begin{abstract}
The metastasis-related molecules CD133, CD44v6 and human tissue factor (TF) have been shown to be associated with tumor invasion and metastasis. This study aimed to determine whether co-expression of these three molecules was associated with metastasis and overall prognosis in pancreatic carcinoma. We analyzed the expression profiles of these three molecules by immunohistochemistry and evaluated the relationship of their expression profiles with metastasis and prognosis in 109 pancreatic carcinomas. The results showed that the expression levels of CD133, CD44v6 and TF were increased in pancreatic carcinoma. Co-expression of CD133, CD44v6 and TF (tri-expression) was also detected in pancreatic carcinoma. Clinical analysis showed that individual expression of CD133, CD44v6 or TF was associated with vessel invasion, lymph node metastasis and liver metastasis, while tri-expression was associated with lymph node metastasis. Survival analysis showed that patients with co-expression of CD133 and TF or tri-expression had lower and the lowest overall survival rates, respectively. Univariate analysis showed that T-factor, lymph node metastasis, TNM stage, and individual levels or tri-expression of CD133, CD44v6 and TF were survival risk factors. Multivariate analysis showed that tri-expression of CD133, CD44v6 and TF was an independent predictor of survival. These results suggest that overexpression of CD133, CD44v6 and TF is associated with pancreatic carcinoma metastasis. Tri-expression of these three molecules may be a useful predictor for pancreatic carcinoma prognosis.
\end{abstract}

Correspondence to: Professor Xiaowu Li, Department of Hepatobiliary Surgery Institute, Southwest Hospital, Third Military Medical University, 30 Gaotanyan Street, Shapingba, Chongqing 400038, P.R. China

E-mail: lixw1966@163.com

*Contributed equally

Key words: CD133, CD44v6, TF, pancreatic carcinoma, metastasis

\section{Introduction}

Pancreatic carcinoma is one of the most aggressive cancers. Early metastasis to regional lymph nodes and finally hematogenous spread to distant organs are the leading causes of the low 5-year survival rate $(1,2)$. Previous studies have shown that various distinct proteins are involved in the different steps of cancer progression. In pancreatic carcinoma, CD133, CD44 and $\mathrm{TF}$ are distinct proteins involved in invasion and metastasis (3-5).

CD133, as an important marker of cancer stem cells (CSCs), has been used in the identification of CSCs from several solid tumors (6-12). A CD $44^{+} / \mathrm{CD} 133^{+} \mathrm{CSC}$ population has been identified that exhibits extensive proliferation, self-renewal, differentiation and invasion $(6,13)$. Recent studies have shown that $\mathrm{CD}_{133}{ }^{+}$circulating tumor cells (CTCs) are believed to be directly involved in the metastatic process of colon cancer (14). In pancreatic carcinoma, CD133 has been shown to be associated with lymph node metastasis (3).

CD44, a transmembrane glycoprotein, has multiple variant isoforms (CD44v2-10) (15,16). CD44 standard (CD44s) can be found in most tissues in the adult organism, including the hematopoietic system, whereas the variant isoforms are expressed in specific epithelial tissues and cancers $(17,18)$. Recent studies have indicated that CD44v6, a metastatic marker, is unregulated in aggressive pancreatic cancer CSC subpopulations, and blockage of CD44v6 suppresses the metastasis of pancreatic carcinoma cells $(19,20)$. There is ample evidence to show that a CD44 isoform, and specifically CD44v6 as an HA binding protein, or a co-receptor for c-Met and VEGFR-2 is crucial for the establishment of primary tumors as well as for metastasis (21). Interaction of CD44 with $\mathrm{P}$-selectin facilitates tumor cell survive in circulation via binding to platelets, thereby making $\mathrm{CD} 44^{+}$prone to be involved in distant metastases (22-24).

Human tissue factor (TF) is the cell surface receptor of factor VII (FVII), which is responsible for triggering blood coagulation $(25,26)$. The expression of $\mathrm{TF}$ in tumors can alter the tumor microenvironment, thus facilitating the survival and metastasis of CSCs (27-30). A strong correlation between TF expression and hepatic metastasis, but not lymph node 
metastasis, has been recognized in colorectal carcinoma patients (31). In pancreatic carcinoma, TF expression occurs preferentially at the invasive front of the tumor and is correlated with angiogenesis, lymph node and liver metastases and a poor prognosis $(5,32)$.

Indeed, recent studies have shown that certain types of cancer cells expressing markers of CSCs (CD133) also exhibit elevated expression of TF or CD44 $(28,30,33,34)$. However, the role of these three molecules in tumor metastasis is unclear. To determine whether tri-expression of these three molecules is associated with metastasis and prognosis in pancreatic carcinoma, we analyzed the expression profiles of these three molecules in pancreatic carcinoma tissues by immunohistochemistry and further evaluated the relationship of their expression profiles with metastasis and prognosis of pancreatic carcinoma.

\section{Materials and methods}

Patients and specimens. A total of 109 patients (71 male and 38 female) with a median age 58 years (range $36-86$ years) underwent surgery at the Department of Hepatobiliary Surgery Institute, Southwest Hospital, Third Military Medical University, China, for pancreatic carcinoma from January 2007 to June 2010. All the patients underwent curative resection by pancreaticoduodenectomy and pylorus-preserving pancreaticoduodenectomy with lymph node dissection. None of the patients had received neoadjuvant or adjuvant radio/ chemotherapy. Formalin-fixed paraffin-embedded samples were obtained for immunohistochemical analysis. The number of patients with pT1, pT2, pT3, and pT4 tumors was 19 (17.4\%), 33 (30.3\%), 53 (48.6\%), and 4 (3.7\%), respectively. Resected primary tumors and lymph nodes were histologically examined by hematoxylin and eosin staining using the TNM (tumor-node-metastasis) classification system. Histologically, all of the tumors were invasive ductal adenocarcinomas $(9$ well-differentiated, 73 moderately differentiated and 27 poorly differentiated). Lymph node metastasis and vascular invasion were observed in 44 (40.4\%) and 37 tumors (33.9\%), respectively. All patients were assessed by radiography, ultrasonography and computed tomography every 3 months after discharge. New lesions detected by imaging were considered indicative of relapse. The median follow-up period was 13 months (range 3-46 months). During this period, 14 patients experienced recurrence of liver disease.

This study was approved by the Ethics Committee of the Southwest Hospital, and all patients provided written informed consent.

Immunohistochemistry. Specimens were fixed in formalin, embedded in paraffin and cut into $3-\mathrm{mm}$ sections. Sections were deparaffinized in xylene, rehydrated in a graded series of ethanol solutions and incubated in 3.0\% hydrogen peroxide in methanol for 30 min to block endogenous peroxidase action. Slides were heated at $120^{\circ} \mathrm{C}$ in an autoclave in $10 \mathrm{mM}$ sodium citrate (pH 6.0) for $130 \mathrm{sec}$ and cooled to room temperature. After blocking with $10 \%$ goat serum for $30 \mathrm{~min}$, the sections were incubated overnight at $4^{\circ} \mathrm{C}$ with primary antibodies for CD133 (rabbit polyclonal; Bioss; bs-0209R, dilution 1:100), CD44v6 (mouse monoclonal; Invitrogen; 33-6700, dilution
1:50) and TF (rabbit polyclonal, Boster; BA1714, dilution 1:100). Negative controls were obtained by omitting the primary antibody. The sections were incubated with peroxidase-conjugated anti-mouse/rabbit immunoglobulins (Dako EnVision $^{\mathrm{TM}}$ System; K5007) for $60 \mathrm{~min}$ at $37^{\circ} \mathrm{C}$. The peroxidase reaction was developed with 3,3'-diaminobenzidine as the chromogen and counterstained with hematoxylin.

Criteria for assessing immunohistochemical results. All the immunostained sections were evaluated independently by two investigators without knowledge of the clinical or pathological backgrounds of the patients. Ten random fields were selected, and expression was evaluated in 1,000 tumor cells (100 cells per field) with an image analyzer (MetaMorph Imaging System version 6.0). The immunohistochemical grade was quantified according to the proportion of stained cells. Specimens were defined as having positive expression if there were tumor cells distinctly stained by anti-CD133, $\mathrm{CD} 44 \mathrm{v} 6$ or TF antibody. The staining intensity of CD133 was scored as $3+(>25 \%), 2+(5-25 \%), 1+(<5 \%)$ or $0(0 \%)$ respectively, according to the percentage of positively stained cells (Fig. 1A, D and G) (3,35). Similarly, the staining intensity of CD44v6 was scored as $3+(>50 \%), 2+(10-50 \%), 1+(<10 \%)$ or $0(0 \%)$, respectively (Fig. 1B, E and H) (36). The staining intensity of TF was scored as $3+(>66 \%), 2+(33-66 \%), 1+(<33 \%)$ or $0(0 \%)$, respectively (Fig. 1C, F and I) (5). For statistical analysis, as well as to reduce intraobserver variability, the immunohistochemical scores were further grouped into two categories: low (grade 0 or $1+$ ) or high (grade $2+$ or $3+$ ).

Statistical analyses. Group differences were statistically analyzed using the $\chi^{2}$ test. The Kaplan-Meier method was used to analyze survival and the log-rank test was used to estimate differences in survival. Prognostic factors were examined using univariate and multivariate analyses (Cox proportional hazards regression model). During the Cox regression, Backward LR method was applied, and values of variables not in the equation were picked from step one. P-values $<0.05$ were considered statistically significant. Statistical analysis was performed using IBM SPSS Statistics 19. All the statistical analyses were completed under the guidance of experienced experts in the Statistics Department.

\section{Results}

Overexpression of CD133, CD44v6 and TF in pancreatic carcinoma. It has been shown that CD133, CD44v6 and TF play important roles in the process of tumor metastasis. To analyze the expression patterns of CD133, CD44v6 and TF in pancreatic carcinoma, we applied immunohistochemistry in 109 pancreatic carcinoma and 8 normal pancreatic samples. As shown in Table I and Fig. 2, compared with the normal pancreatic tissues, the expression of CD133 in the pancreatic carcinoma was low in 44 samples and high in 65 samples. Similarly, the expression of CD44v6 was low in 59 samples and high in 50 samples. Additionally, the expression of TF was low in 42 samples and high in 67 samples. Furthermore, 46/109 pancreatic carcinoma samples showed tri-expression of CD133, CD44v6 and TF and an additional 16/109 samples showed bi-expression of CD133 and TF but were CD44v6 

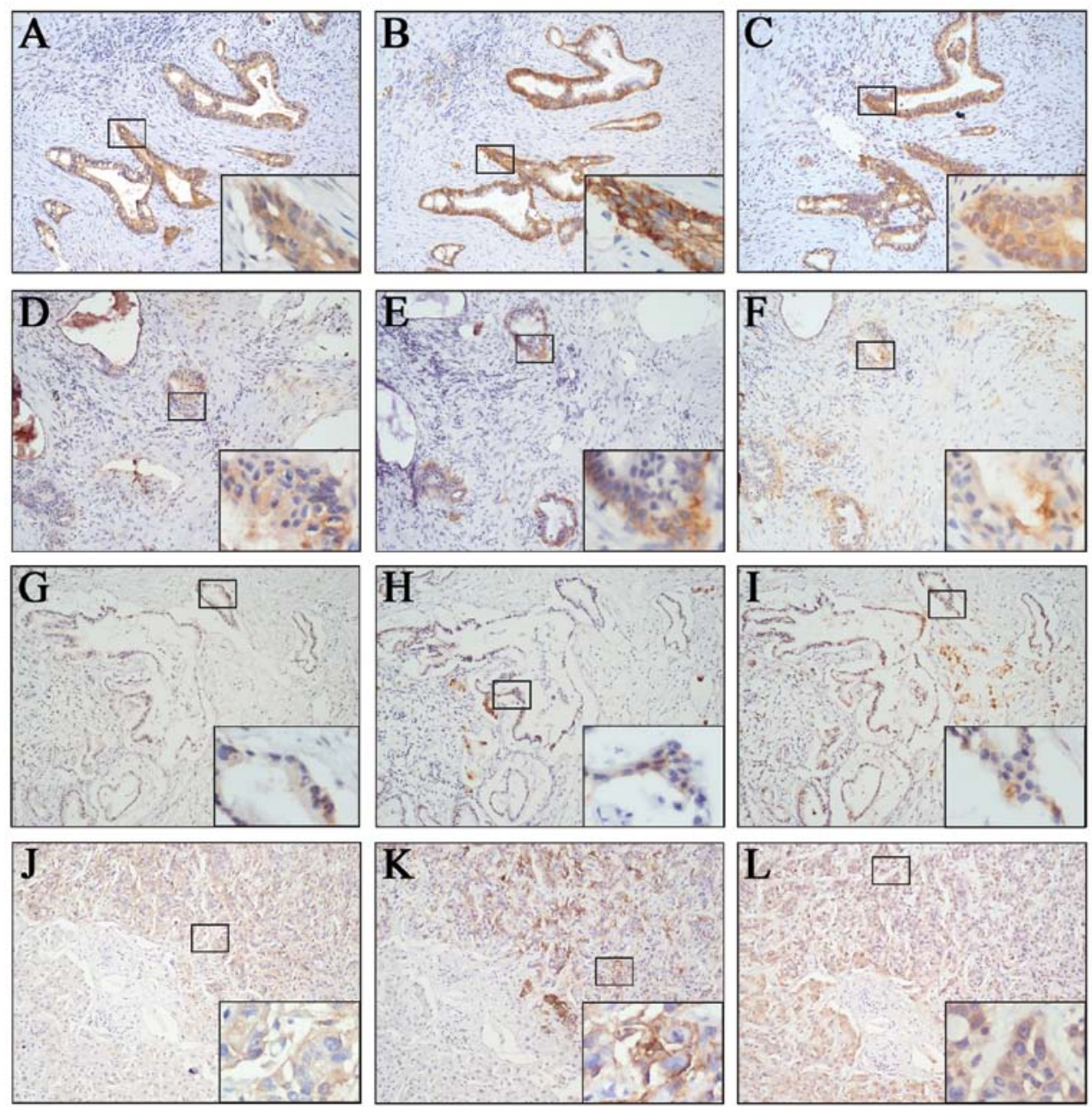

Figure 1. Immunohistochemical staining of CD133, CD44v6 and TF in pancreatic carcinoma. (A-I) Samples from primary pancreatic carcinoma. (J-L) Liver samples from metastatic pancreatic carcinoma. (A, D and G) are CD133-positive (3+), CD133-positive (2+) and CD133-positive (1+), respectively. (B, E and $\mathrm{H}$ ) are CD44v6-positive (3+), CD44v6-positive (2+) and CD44v6-positive (1+), respectively. (C, F and I) are TF-positive (3+), TF-positive (2+) and TF-positive (1+), respectively. (J, K and L) are CD133-positive (3+), CD44v6-positive (3+) and TF-positive (3+), respectively. Original magnification, x100; $\mathrm{x} 400$ magnification is shown in the bottom right box.

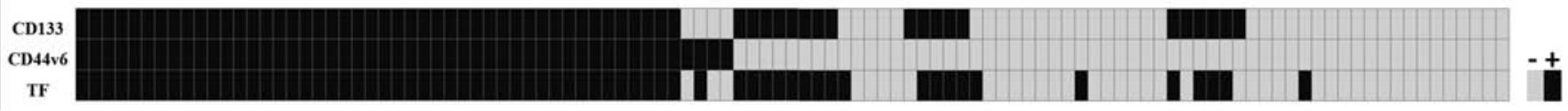

Figure 2. Schematic figure of the expression level of CD133, CD44v6 and TF. Each column represent a sample of pancreatic carcinoma (109 samples). The expression level is represented by the depth of shading: "-": low and "+": high.

low. Of the 46 tri-expression samples, 11 samples (23.9\%) had hepatic metastases. However, of the 16 bi-expression samples, only 1 sample (7.1\%) had hepatic metastasis (data not shown). These results suggest that the expression of CD133, CD44v6 and $\mathrm{TF}$ is increased in pancreatic carcinoma and tri-expression of these three molecules may be required for distant metastasis of pancreatic carcinoma.

Expression levels of CD133, CD44v6 and TF are correlated with vascular invasion and lymph node and liver metastases. To investigate whether CD133, CD44v6 and TF are involved in the metastasis of pancreatic carcinoma, we further analyzed the relationship between the clinical characteristics of the pancreatic carcinoma patients and the expression levels of CD133,
Table I. Expression of CD133, CD44v6, TF and their coexpression in the pancreatic carcinoma samples $(n=109)$.

\begin{tabular}{lcc}
\hline Variables & \multicolumn{2}{c}{ Expression level } \\
\cline { 2 - 3 } & Low & High \\
\hline CD133 & 44 & 65 \\
CD44v6 & 59 & 50 \\
TF & 42 & 67 \\
CD133 + CD44v6 & & 0 \\
CD133 + TF & & 16 \\
CD44v6 + TF & & 1 \\
CD133 + CD44v6 + TF & & 46
\end{tabular}


Table II. Clinicopathological parameters and immunohistochemical labeling of CD44v6, CD133 and TF ( $\mathrm{n}=109)$.

\begin{tabular}{|c|c|c|c|c|c|c|c|c|c|}
\hline \multirow[b]{2}{*}{ Variables } & \multicolumn{3}{|c|}{ CD133 } & \multicolumn{3}{|c|}{ CD44v6 } & \multicolumn{3}{|c|}{$\mathrm{TF}$} \\
\hline & $\begin{array}{l}\text { High } \\
\text { n (\%) }\end{array}$ & $\begin{array}{l}\text { Low } \\
\mathrm{n}(\%)\end{array}$ & $\begin{array}{l}\text { P-value } \\
\chi^{2} \text {-value }\end{array}$ & $\begin{array}{l}\text { High } \\
\text { n (\%) }\end{array}$ & $\begin{array}{l}\text { Low } \\
\text { n (\%) }\end{array}$ & $\begin{array}{l}\text { P-value } \\
\chi^{2} \text {-value }\end{array}$ & $\begin{array}{l}\text { High } \\
\text { n (\%) }\end{array}$ & $\begin{array}{l}\text { Low } \\
\text { n (\%) }\end{array}$ & $\begin{array}{l}\text { P-value } \\
\chi^{2} \text {-value }\end{array}$ \\
\hline Gender & & & 0.583 & & & 0.527 & & & 0.498 \\
\hline Female & $24(36.9)$ & $14(31.8)$ & 0.301 & $19(38.0)$ & $19(32.2)$ & 0.400 & $25(37.3)$ & $13(31.0)$ & 0.460 \\
\hline Male & $41(63.1)$ & $30(68.2)$ & & $31(62.0)$ & $40(67.8)$ & & $42(62.7)$ & $29(69.0)$ & \\
\hline Age, years & & & 0.962 & & & 0.743 & & & 0.526 \\
\hline$<65$ & $47(72.3)$ & $32(72.7)$ & 0.002 & $37(74.0)$ & $42(71.2)$ & 0.107 & $50(74.6)$ & $29(69.0)$ & 0.403 \\
\hline$\geq 65$ & $18(27.7)$ & $12(27.3)$ & & $13(26.0)$ & $17(28.8)$ & & $17(25.4)$ & $13(31.0)$ & \\
\hline Tumor location & & & 0.083 & & & 0.237 & & & 0.112 \\
\hline Head & $53(81.5)$ & $41(93.2)$ & 2.997 & $41(82.0)$ & $53(89.8)$ & 1.398 & $55(82.1)$ & 39 (92.9) & 2.522 \\
\hline Body/Tail & $12(18.5)$ & $3(6.8)$ & & $9(18.0)$ & $6(10.2)$ & & $12(17.9)$ & $3(7.1)$ & \\
\hline Tumor size, cm & & & 0.908 & & & 0.805 & & & 0.702 \\
\hline$\leq 2$ & $20(30.8)$ & $14(31.8)$ & 0.013 & $15(30.0)$ & $19(32.2)$ & 0.061 & $20(29.9)$ & $14(33.3)$ & 0.146 \\
\hline$>2$ & $45(69.2)$ & $30(68.2)$ & & $35(70.0)$ & $40(67.8)$ & & $47(70.1)$ & $28(66.7)$ & \\
\hline Lymph node status & & & 0.002 & & & 0.002 & & & 0.001 \\
\hline Negative & $31(47.7)$ & $34(77.3)$ & 9.538 & $22(44.0)$ & $43(72.9)$ & 9.378 & $32(47.8)$ & 33 (78.6) & 10.181 \\
\hline Positive & $34(52.3)$ & $10(22.7)$ & & $28(56.0)$ & $16(27.1)$ & & $35(52.2)$ & $9(21.4)$ & \\
\hline Vascular invasion & & & 0.004 & & & 0.041 & & & 0.000 \\
\hline Negative & $36(55.4)$ & $36(81.8)$ & 8.177 & $28(56.0)$ & $44(74.6)$ & 4.165 & $35(52.2)$ & $37(88.1)$ & 14.803 \\
\hline Positive & $29(44.6)$ & 8 (18.2) & & $22(44.0)$ & $15(25.4)$ & & $32(47.8)$ & $5(11.9)$ & \\
\hline Neural invasion & & & 0.261 & & & 0.302 & & & 0.792 \\
\hline Negative & $41(63.1)$ & $23(52.3)$ & 1.264 & $32(64.0)$ & $32(54.2)$ & 1.064 & $40(59.7)$ & $24(57.1)$ & 0.070 \\
\hline Positive & $24(36.9)$ & $21(47.7)$ & & $18(36.0)$ & $27(45.8)$ & & $27(40.3)$ & $18(42.9)$ & \\
\hline Duodenal invasion & & & 0.588 & & & 0.28 & & & 0.881 \\
\hline Negative & $52(80.0)$ & $37(84.1)$ & 0.293 & $43(86.0)$ & $46(78.0)$ & 1.166 & $55(82.1)$ & $34(81.0)$ & 0.022 \\
\hline Positive & $13(20.0)$ & 7 (15.9) & & $7(14.0)$ & $13(22.0)$ & & $12(17.9)$ & $8(19.0)$ & \\
\hline Hepatic metastases & & & 0.033 & & & 0.003 & & & 0.046 \\
\hline Negative & $53(81.5)$ & $42(95.5)$ & 4.539 & $39(76.5)$ & $56(96.0)$ & 8.728 & $55(82.1)$ & $40(95.2)$ & 3.987 \\
\hline Positive & $12(18.5)$ & $2(4.5)$ & & $12(23.5)$ & $2(3.4)$ & & $12(17.9)$ & $2(4.8)$ & \\
\hline Differentiation & & & 0.168 & & & 0.025 & & & 0.298 \\
\hline Poor & $20(30.8)$ & $7(15.9)$ & 3.566 & $18(36.0)$ & $9(15.3)$ & 7.400 & $20(29.9)$ & $7(16.7)$ & 2.421 \\
\hline Moderate & $41(63.1)$ & $32(72.7)$ & & $30(60.0)$ & $43(72.9)$ & & $42(62.7)$ & $31(73.8)$ & \\
\hline Well & $4(6.2)$ & $5(11.4)$ & & $2(4.0)$ & 7 (11.9) & & $5(7.5)$ & $4(9.5)$ & \\
\hline T-factor (UICC) & & & 0.388 & & & 0.671 & & & 0.306 \\
\hline $\mathrm{T} 1$ & $11(16.9)$ & $8(18.2)$ & 3.023 & $9(18.0)$ & $10(16.9)$ & 1.549 & $12(17.9)$ & $7(16.7)$ & 3.614 \\
\hline $\mathrm{T} 2$ & $16(24.6)$ & $17(38.6)$ & & $14(28.0)$ & $19(32.2)$ & & $16(23.9)$ & $17(40.5)$ & \\
\hline $\mathrm{T} 3$ & $35(53.8)$ & $18(40.9)$ & & $24(48.0)$ & $29(49.2)$ & & $36(53.7)$ & $17(40.5)$ & \\
\hline $\mathrm{T} 4$ & $3(4.6)$ & $1(2.3)$ & & $3(6.0)$ & 1 (1.7) & & $3(4.5)$ & $1(2.4)$ & \\
\hline Stage (UICC) & & & 0.002 & & & 0.004 & & & 0.001 \\
\hline $1 \mathrm{~A}$ & $3(4.6)$ & 7 (15.9) & 19.132 & $2(4.0)$ & $8(13.6)$ & 17.479 & $3(4.5)$ & 7 (16.7) & 20.563 \\
\hline 1B & $7(10.8)$ & $14(31.8)$ & & $6(12.0)$ & $15(25.4)$ & & 7 (10.4) & $14(33.3)$ & \\
\hline $2 \mathrm{~A}$ & $14(21.5)$ & $12(27.3)$ & & $8(16.0)$ & $18(30.5)$ & & $15(22.4)$ & $11(26.2)$ & \\
\hline $2 \mathrm{~B}$ & $27(41.5)$ & 8 (18.2) & & $21(42.0)$ & $14(23.7)$ & & $28(41.8)$ & 7 (16.7) & \\
\hline 3 & 1 (1.5) & $1 \quad(2.3)$ & & $1(2.0)$ & 1 (1.7) & & 1 (1.5) & $1(2.4)$ & \\
\hline 4 & $13(20.0)$ & $2(4.5)$ & & $12(24.0)$ & $3(5.1)$ & & $13(19.4)$ & $2(4.8)$ & \\
\hline
\end{tabular}

Bold values indicate P-values <0.05. UICC, Union for International Cancer Control. 
Table III. Clinicopathological parameters and immunohistochemical labeling of bi-expression of CD133 + TF and tri-expression of CD133, CD44v6 and TF (n=109).

\begin{tabular}{|c|c|c|c|c|c|c|}
\hline \multirow[b]{2}{*}{ Variables } & \multicolumn{3}{|c|}{$\mathrm{CD} 133+\mathrm{TF}$} & \multicolumn{3}{|c|}{$\mathrm{CD} 44 \mathrm{v} 6+\mathrm{CD} 133+\mathrm{TF}$} \\
\hline & $\begin{array}{l}\text { High } \\
\text { n (\%) }\end{array}$ & $\begin{array}{l}\text { Low } \\
\text { n (\%) }\end{array}$ & $\begin{array}{l}\text { P-value } \\
\chi^{2} \text {-value }\end{array}$ & $\begin{array}{l}\text { High } \\
\text { n (\%) }\end{array}$ & $\begin{array}{l}\text { Low } \\
\mathrm{n}(\%)\end{array}$ & $\begin{array}{l}\text { P-value } \\
\chi^{2} \text {-value }\end{array}$ \\
\hline Gender & & & 0.574 & & & 0.228 \\
\hline Female & $23(37.1)$ & $15(31.9)$ & 0.316 & $19(41.3)$ & $19(30.2)$ & 1.454 \\
\hline Male & $39(62.9)$ & $32(68.1)$ & & $27(58.7)$ & $44(69.8)$ & \\
\hline Age, years & & & 0.645 & & & 0.774 \\
\hline$<65$ & $46(74.2)$ & $33(70.2)$ & 0.212 & $34(73.9)$ & $45(71.4)$ & 0.082 \\
\hline$\geq 65$ & $16(25.8)$ & $14(29.8)$ & & $12(26.1)$ & $18(28.6)$ & \\
\hline Tumor location & & & 0.166 & & & 0.133 \\
\hline Head & $51(82.3)$ & $43(91.5)$ & 1.920 & $37(80.4)$ & $57(90.5)$ & 2.259 \\
\hline Body/Tail & $11(17.7)$ & $4(8.5)$ & & $9(19.6)$ & $6 \quad(9.5)$ & \\
\hline Tumor size, $\mathrm{cm}$ & & & 0.576 & & & 0.572 \\
\hline$\leq 2$ & $18(29.0)$ & $16(34.0)$ & 0.313 & $13(28.3)$ & $21(33.3)$ & 0.319 \\
\hline$>2$ & $44(71.0)$ & $31(66.0)$ & & $33(71.7)$ & $42(66.7)$ & \\
\hline Lymphatic invasion & & & 0.000 & & & 0.000 \\
\hline Negative & $28(45.2)$ & $37(78.7)$ & 12.510 & $18(39.1)$ & $47(74.6)$ & 13.898 \\
\hline Positive & $34(54.8)$ & $10(21.3)$ & & $28(60.9)$ & $16(25.4)$ & \\
\hline Vascular invasion & & & 0.001 & & & 0.027 \\
\hline Negative & $33(53.2)$ & $39(83.0)$ & 10.555 & $25(54.3)$ & 47 (74.6) & 4.865 \\
\hline Positive & $29(46.8)$ & $8(17.0)$ & & $21(45.7)$ & $16(25.4)$ & \\
\hline Neural invasion & & & 0.308 & & & 0.433 \\
\hline Negative & 39 (62.9) & $25(53.2)$ & 1.040 & $29(63.0)$ & $35(55.6)$ & 0.615 \\
\hline Positive & $23(37.1)$ & $22(46.8)$ & & $17(37.0)$ & $28(44.4)$ & \\
\hline Duodenal invasion & & & 0.755 & & & 0.470 \\
\hline Negative & $50(80.6)$ & $39(83.0)$ & 0.097 & $39(84.8)$ & $50(79.4)$ & 0.521 \\
\hline Positive & $12(19.4)$ & $8(17.0)$ & & $7(15.2)$ & $13(20.6)$ & \\
\hline Hepatic metastases & & & 0.020 & & & 0.003 \\
\hline Negative & $50(80.6)$ & 45 (95.7) & 5.445 & $35(76.1)$ & $60(95.2)$ & 8.711 \\
\hline Positive & $12(19.4)$ & $2(4.3)$ & & $11(23.9)$ & $3(4.8)$ & \\
\hline Differentiation & & & 0.105 & & & 0.022 \\
\hline Poor & $20(32.3)$ & 7 (14.9) & 4.515 & $17(37.0)$ & $10(15.9)$ & 7.672 \\
\hline Moderate & $38(61.3)$ & $35(74.5)$ & & $25(54.3)$ & $46(73.0)$ & \\
\hline Well & $4(6.5)$ & $5(10.6)$ & & $2(4.3)$ & 7 (11.1) & \\
\hline T-factor (UICC) & & & 0.522 & & & 0.381 \\
\hline $\mathrm{T} 1$ & $10(16.1)$ & $9(19.1)$ & 2.250 & $9(19.6)$ & $10(15.9)$ & 3.067 \\
\hline $\mathrm{T} 2$ & $16(25.8)$ & $17(36.2)$ & & $11(23.9)$ & $22(34.9)$ & \\
\hline $\mathrm{T} 3$ & $33(53.2)$ & $20(42.6)$ & & $23(50.0)$ & $30(47.6)$ & \\
\hline $\mathrm{T} 4$ & $3(4.8)$ & 1 (2.1) & & $3(6.5)$ & 1 (1.6) & \\
\hline Stage (UICC) & & & 0.000 & & & 0.000 \\
\hline $1 \mathrm{~A}$ & $2(3.2)$ & $8(17.0)$ & 22.836 & $2(4.3)$ & 8 (12.7) & 28.584 \\
\hline 1B & 7 (11.3) & $14(29.8)$ & & $3(6.5)$ & $18(28.6)$ & \\
\hline $2 \mathrm{~A}$ & $12(19.4)$ & $14(29.8)$ & & $7(15.2)$ & $19(30.2)$ & \\
\hline $2 \mathrm{~B}$ & $27(43.5)$ & $8(17.0)$ & & $27(58.7)$ & $14(22.2)$ & \\
\hline 3 & 1 (1.6) & $1 \quad(2.1)$ & & $1 \quad(2.2)$ & 1 (1.6) & \\
\hline 4 & $13(21.0)$ & $2(4.3)$ & & $12(26.1)$ & $3(4.8)$ & \\
\hline
\end{tabular}

Bold values indicate P-values $<0.05$. UICC, Union for International Cancer Control. 
Table IV. Median survival and 1-, 2- and 3-year survival rates and CD133, CD44v6, TF and their co-expression.

\begin{tabular}{|c|c|c|c|c|c|c|c|c|c|c|}
\hline \multirow[b]{2}{*}{ Variables } & \multicolumn{2}{|c|}{ CD133 } & \multicolumn{2}{|c|}{ CD44v6 } & \multicolumn{2}{|c|}{$\mathrm{TF}$} & \multicolumn{2}{|c|}{$\mathrm{CD} 133+\mathrm{TF}$} & \multicolumn{2}{|c|}{$\begin{array}{c}\text { CD44v6 + } \\
\text { CD133 + TF }\end{array}$} \\
\hline & Low & High & Low & High & Low & High & Low & High & Low & High \\
\hline Median survival time (months) & 14 & 11 & 13 & 9 & 13 & 11 & 14 & 11 & 14 & 9 \\
\hline 1-year survival rate $(\%)$ & 67.6 & 41.9 & 55.6 & 31.0 & 56.8 & 35.8 & 59.0 & 33.2 & 58.1 & 25.3 \\
\hline 2-year survival rate $(\%)$ & 25.5 & 12.7 & 24.6 & 10.3 & 25.5 & 12.6 & 26.8 & 11.1 & 25.0 & 8.4 \\
\hline 3-year survival rate $(\%)$ & 11.3 & 3.2 & 8.9 & 3.9 & 14.2 & 0.0 & 8.0 & 0.0 & 10.4 & 0.0 \\
\hline
\end{tabular}

Table V. Univariate and multivariate Cox regression of prognostic factors for overall survival in pancreatic adenocarcinoma.

\begin{tabular}{|c|c|c|c|}
\hline \multirow[b]{2}{*}{ Independent factors } & \multirow{2}{*}{$\frac{\text { Univariate } \mathrm{P}}{\mathrm{P} \text {-value }}$} & \multicolumn{2}{|c|}{ Multivariate $\mathrm{P}$} \\
\hline & & HR $(95 \% \mathrm{CI})$ & P-value \\
\hline Age (years <65/ $\geq 65$ ) & 0.281 & & \\
\hline Gender (female/male) & 0.944 & & \\
\hline Tumor size $(<2 / \geq 2 \mathrm{~cm})$ & 0.860 & & \\
\hline pN (negative/positive) & 0.000 & $1.642(1.009-2.674)$ & 0.046 \\
\hline Pv (negative/positive) & 0.024 & $1.354(0.646-2.835)$ & 0.422 \\
\hline Hepatic metastases (negative/positive) & 0.028 & $1.190(0.430-3.293)$ & 0.737 \\
\hline Differentiation (poor/moderate/well) & 0.672 & & \\
\hline pT $(\mathrm{T} 1,2 / \mathrm{T} 3,4)$ & 0.017 & $0.984(0.471-2.058)$ & 0.966 \\
\hline pStage (I,II/III,IV) & 0.000 & $1.652(1.081-2.524)$ & 0.020 \\
\hline CD44v6 expression (low/high) & 0.009 & $0.968(0.278-3.372)$ & 0.960 \\
\hline CD133 expression (low/high) & 0.013 & $1.008(0.227-4.486)$ & 0.992 \\
\hline TF expression (low/high) & 0.018 & $0.726(0.165-3.194)$ & 0.672 \\
\hline CD133 + TF expression (low/high) & 0.005 & $1.305(0.149-11.435)$ & 0.810 \\
\hline CD44v6 + CD133 + TF expression (low/high) & 0.000 & $1.774(1.102-2.854)$ & 0.018 \\
\hline
\end{tabular}

Bold values indicate P-values $<0.05$. HR, hazard ratio; $\mathrm{CI}$, confidence interval; $\mathrm{pN}$, pathological node stage; $\mathrm{pV}$, pathological vessel status; $\mathrm{pT}$, T-factor; pStage, TNM stage (UICC).

CD44v6 and TF. As shown in Table II, overexpression of CD133 was correlated with vascular invasion $\left(\mathrm{P}=0.004, \chi^{2}=8.177\right)$, lymph node metastasis $\left(\mathrm{P}=0.002, \chi^{2}=9.538\right)$, hepatic metastasis $\left(\mathrm{P}=0.033, \chi^{2}=4.539\right)$ and $\mathrm{TNM}$ stage $\left(\mathrm{P}=0.002, \chi^{2}=19.132\right)$. Overexpression of CD44v6 was correlated with vascular invasion $\left(\mathrm{P}=0.041, \chi^{2}=4.165\right)$, lymph node metastasis $(\mathrm{P}=0.002$, $\left.\chi^{2}=9.378\right)$, hepatic metastasis $\left(\mathrm{P}=0.003, \chi^{2}=8.728\right)$ and TNM stage $\left(\mathrm{P}=0.004, \chi^{2}=17.479\right)$. Overexpression of TF was correlated with vascular invasion $\left(\mathrm{P}=0.000, \chi^{2}=14.803\right)$, lymph node metastasis $\left(\mathrm{P}=0.001, \chi^{2}=10.181\right)$, hepatic metastasis $(\mathrm{P}=0.046$, $\left.\chi^{2}=3.987\right)$ and TNM stage $\left(\mathrm{P}=0.001, \chi^{2}=20.563\right)$. As shown in Table III, co-expression of CD133 and TF was correlated with vascular invasion $\left(\mathrm{P}=0.001, \chi^{2}=10.555\right)$, lymph node metastasis $\left(\mathrm{P}=0.000, \chi^{2}=12.510\right)$ and hepatic metastasis $(\mathrm{P}=0.020$, $\left.\chi^{2}=5.445\right)$. The tri-expression of CD133, CD44v6 and TF was correlated with vascular invasion $\left(\mathrm{P}=0.027, \chi^{2}=4.865\right)$, lymph node metastasis $\left(\mathrm{P}=0.000, \chi^{2}=13.898\right)$, hepatic metastasis $\left(\mathrm{P}=0.003, \chi^{2}=8.711\right)$ and TNM stage $(\mathrm{P}=0.000$, $\chi^{2}=28.584$ ), but showed greater differences in metastases to the lymph nodes and the liver. Notably, further analysis found that tri-expression and bi-expression had similar rates of lymph node metastasis and vascular invasion. Yet, in the 46 tri-expression samples, 11 samples (23.9\%) had hepatic metastases. In contrast, in the 14 bi-expression samples, only 1 sample (7.1\%) had hepatic metastasis. These results indicate that the expression levels of CD133, CD44v6 and TF are correlated with vascular invasion, lymph node metastasis and hepatic metastasis in pancreatic carcinoma and the co-expression of these three molecules in pancreatic carcinoma may imply a poorer prognosis.

Individual expression or tri-expression of the three molecules indicates a poor prognosis in pancreatic carcinoma patients. We further analyzed whether CD133, CD44v6 and TF expression levels affect the 1-year survival rate, median survival time and overall survival of pancreatic carcinoma patients as analyzed using Kaplan-Meier survival analyses. As shown in Table IV, patients with overexpression of CD133 had lower 

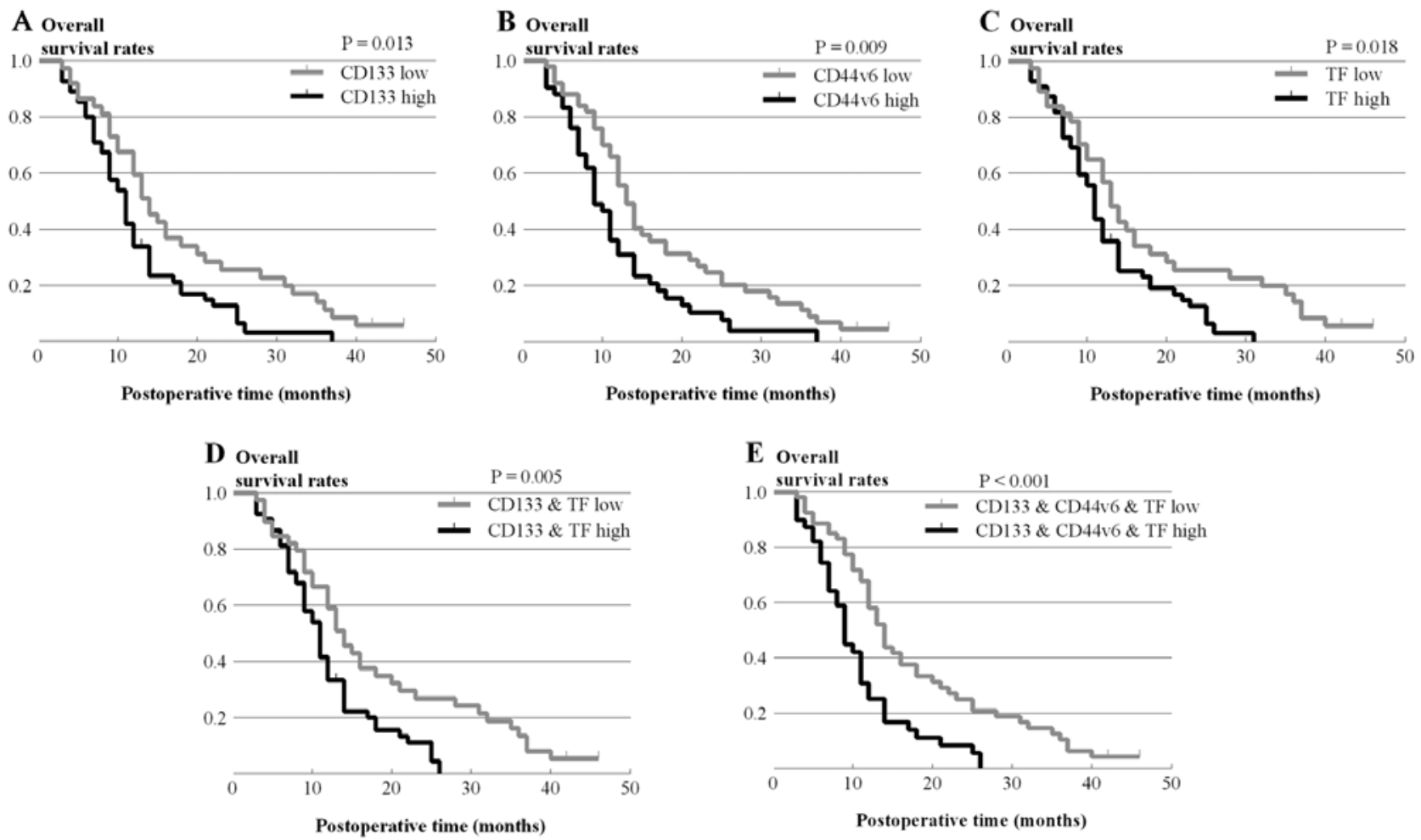

Figure 3. Kaplan-Meier survival curves for overall survival of patients with pancreatic carcinoma according to the expression status of (A) CD133, (B) CD44v6, (C) TF, (D) bi-expression of CD133 and TF, (E) tri-expression of CD133, CD44v6 and TF.

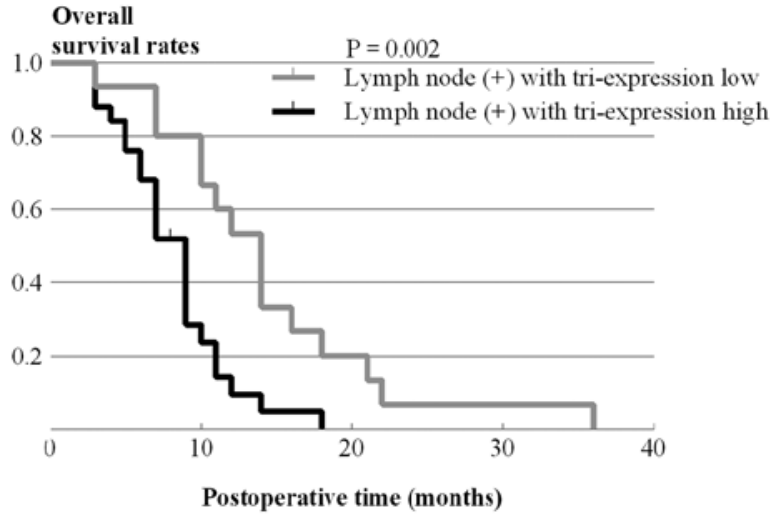

Figure 4. Kaplan-Meier survival curve of patients with lymph node metastasis stratified by tri-expression low vs. tri-expression high.

1-year, 2-year and 3-year survival rates and a shorter median survival time than patients with low expression of CD133 (67.6 vs. $41.9 \%, 25.5$ vs. $12.7 \%, 11.3$ vs. $3.2 \%$ and 14 vs. 11 months, respectively). Patients with overexpression of two or three molecules had even lower survival rates and shorter median survival times.

Kaplan-Meier survival curves showed that the individual overexpression of CD133, CD44v6 or TF significantly decreased overall survival $\left(\mathrm{P}=0.013, \chi^{2}=6.217 ; \mathrm{P}=0.009\right.$, $\chi^{2}=6.756 ; \mathrm{P}=0.018, \chi^{2}=5.622$, respectively) (Fig. 3A-C). Additionally, co-expression of CD133 and TF was also associated with lower overall survival $\left(\mathrm{P}=0.005, \chi^{2}=7.964\right)$ (Fig. 3D). Furthermore, patients with overexpression of all three molecules had the lowest overall survival $\left(\mathrm{P}=0.001, \chi^{2}=12.021\right)$
(Fig. 3E). These data suggest that individual expression or co-expression of these three molecules indicate a poorer prognosis in pancreatic carcinoma patients.

Co-expression of CD133, CD44v6 and TF is an independent predictor of survival in pancreatic carcinoma patients. To further identify the independent risk factors of survival, we applied univariate and multivariate analyses to investigate the survival rate for pancreatic adenocarcinoma. In the univariate analysis, we found that age, gender and differentiation were not risk factors of survival. However, T-factor (pT), lymph node metastasis $(\mathrm{pN})$, vascular invasion $(\mathrm{pV})$, TNM stage and individual expression or co-expression of CD133, CD44v6 and TF were all risk factors for survival (Table V). Multivariate analysis showed that lymph node metastasis $(\mathrm{pN})$ and TNM stage were independent predictors of survival rate $(\mathrm{P}=0.046$ and $\mathrm{P}=0.020$, respectively). Yet, the individual expression levels of CD133, CD44v6 and TF were not independent predictors. Importantly, the co-expression of CD133, CD44v6 and TF was also found to be an independent predictor of survival $(\mathrm{P}=0.018)$ (Table V). Therefore, although CD133, CD44v6 and TF all had an effect on the survival rate, only co-expression of CD133, CD44v6 and TF was found to be an independent predictor of survival in pancreatic carcinoma patients.

\section{Discussion}

We first characterized the expression patterns of three tumor metastasis-related molecules, CD44v6, CD133 and TF, in 109 pancreatic carcinoma samples using immunohistochemistry. In this study, we applied antibodies that specifically reacted with CD44-v5, v6 and v7/v8, respectively. We found that, 
except for CD44v6, the other CD44 variants were all negative in the pancreatic carcinoma samples (data not shown). Therefore, among the different splice variants of CD44, CD44v6 is most likely a reliable marker in pancreatic carcinoma (20). Our study showed that the individual expression levels of CD44v6, CD133 and TF were increased in pancreatic carcinoma compared with normal pancreas. These results are consistent with previous studies that have shown that CD44v6, $\mathrm{CD} 133$ and TF are increased in pancreatic carcinoma and are associated with metastasis $(3,32,37)$.

CD133, CD44v6 and TF have been shown to play a very important role in the process of metastasis $(3,37,38)$. CD133 and CD44v6 are markers of CSCs $(11,39,40)$. Previous studies have shoen that a subset of CSCs with $\mathrm{CD} 133^{+} / \mathrm{CD} 44^{+}$has stronger invasive and metastatic capabilities $(33,41)$. TF is unregulated in many cancer cells and impacts tumor progression by altering the tumor microenvironment $(27,28,42)$. Various recent reports have shown that either cancer cells with increased expression of TF or cancer cells with the CSC marker CD133 are important in cancer progression $(43,44)$. However, the relationship between tri-expression of $\mathrm{CD} 133^{+} / \mathrm{CD}_{4} 4^{+} / \mathrm{TF}^{+}$ and tumor progression are still unknown. In the present study, we found that tri-expression of CD133, CD44v6 and TF may be involved in the metastasis of pancreatic carcinoma. First, 46/109 pancreatic carcinoma samples showed tri-expression of CD133, CD44v6 and TF and an additional 16/109 samples showed bi-expression of CD133 and TF but were CD44v6 low. Of the 46 tri-expression samples, 11 (23.9\%) presented with hepatic metastasis. However, of the 14 bi-expression samples, only $1(7.1 \%)$ presented with hepatic metastasis, suggesting that tri-expression of these three molecules may be associated with distant metastasis of pancreatic carcinoma. Indeed, we found strong tri-expression of these three molecules in liver samples from metastasis of pancreatic carcinoma (Fig. 1J-L). Furthermore, the tri-expression of these three molecules showed stronger association with tumor metastasis compared with each molecule individually. These findings may suggest that TF probably alters the tumor microenvironment and facilitates survival and metastasis of pancreatic carcinoma $\mathrm{CD} 133^{+} / \mathrm{CD} 44 \mathrm{v}^{+} \mathrm{CSC}$. Therefore, tri-expression of these three molecules may imply a poor prognosis for pancreatic carcinoma patients.

It has been shown that many invasion-related molecules are implicated in the prognosis of pancreatic carcinoma. In our study, univariate analysis showed that T-factor ( $\mathrm{pT}$ ), lymph node metastasis $(\mathrm{pN})$, vascular invasion $(\mathrm{pV}), \mathrm{TNM}$ stage, individual CD133, CD44v6 and TF expression levels, bi-expression and tri-expression were all significantly correlated with patient survival. In this study, although bi-expression, tri-expression and individual CD133, CD44v6 and TF expression showed similar patient survival curves, bi-expression and tri-expression were associated with reduced survival times in comparison with individual CD133, CD44v6 and TF expression. The "heat map' of expression indicated that these three markers were frequently co-expressed and that bi-expression or even uniexpression were uncommon, suggesting that tri-expression of these three markers may be more important in the prognosis of pancreatic carcinoma. Consistent with the above results, multivariate analysis also showed that tri-expression was an independent prognostic factor. Therefore, tri-expression of
CD133, CD44v6 and TF has significant predictive value for overall survival and a poor prognosis. Therefore, we believe that tri-expression is important in predicting prognosis in pancreatic carcinoma patients. In fact, among patients with lymph node metastasis, patients with tri-expression tumors had a markedly poorer prognosis $(\mathrm{P}=0.002)$ (Fig. 4). Thus, our findings suggest that tri-expression of these three molecules correlates with the aggressiveness of the pancreatic carcinoma. Taken together, we demonstrated that three tumor metastasis-related molecules, CD133, CD44v6 and TF were overexpressed in pancreatic carcinoma and were associated with tumor metastasis and we initially detected the internal relationships among these three molecules in pancreatic carcinoma tissues. Tri-expression of these three molecules may be a useful predictor for pancreatic carcinoma prognosis. This novel finding may provide insight into a novel metastatic mechanism for pancreatic carcinoma.

\section{Acknowledgements}

This study was supported by the Chongqing Natural Science Fund Project (no. 2012JB1032), the Project of the National Natural Science Fund (nos. 81272363, 30872497), the National 863 Project of China (no. 2012AA02A201), the National Basic Research Program of China (973 Program) (no. 2010CB529400), and the National Science and Technology Major Project of China (no. 2008ZX10002-026). We thank Dr Yazhou Wu and Dr Lin Liu of the Statistics Department, Military Preventive Medicine, Third Military Medical University.

\section{References}

1. Wolfgang CL, Herman JM, Laheru DA, et al: Recent progress in pancreatic cancer. CA Cancer J Clin 63: 318-348, 2013.

2. Maeda S, Shinchi H, Kurahara H, et al: Clinical significance of midkine expression in pancreatic head carcinoma. Br J Cancer 97: 405-411, 2007.

3. Maeda S, Shinchi H, Kurahara H, et al: CD133 expression is correlated with lymph node metastasis and vascular endothelial grow th factor-C expression in pancreatic cancer. Br J Cancer 98: 1389-1397, 2008

4. Hong SP, Wen J, Bang S, Park S and Song SY: CD44-positive cells are responsible for gemcitabine resistance in pancreatic cancer cells. Int J Cancer 125: 2323-2331, 2009.

5. Khorana AA, Ahrendt SA, Ryan CK, et al: Tissue factor expression, angiogenesis, and thrombosis in pancreatic cancer. Clin Cancer Res 13: 2870-2875, 2007.

6. Collins AT, Berry PA, Hyde C, Stower MJ and Maitland NJ: Prospective identification of tumorigenic prostate cancer stem cells. Cancer Res 65: 10946-10951, 2005.

7. Ferrandina G, Bonanno G, Pierelli L, et al: Expression of CD133-1 and CD133-2 in ovarian cancer. Int J Gynecol Cancer 18: 506-514, 2008.

8. Florek M, Haase M, Marzesco AM, et al: Prominin-1/CD133, a neural and hematopoietic stem cell marker, is expressed in adult human differentiated cells and certain types of kidney cancer. Cell Tissue Res 319: 15-26, 2005.

9. Ieta K, Tanaka F, Haraguchi N, et al: Biological and genetic characteristics of tumor-initiating cells in colon cancer. Ann Surg Oncol 15: 638-648, 2008.

10. Ma S, Chan KW, Hu L, et al: Identification and characterization of tumorigenic liver cancer stem/progenitor cells. Gastroenterology 132: 2542-2556, 2007.

11. Singh SK, Clarke ID, Terasaki M, et al: Identification of a cancer stem cell in human brain tumors. Cancer Res 63: 5821-5828, 2003.

12. Singh SK, Hawkins C, Clarke ID, et al: Identification of human brain tumour initiating cells. Nature 432: 396-401, 2004. 
13. Haraguchi N, Ohkuma M, Sakashita H, et al: $\mathrm{CD} 133^{+} \mathrm{CD} 44^{+}$ population efficiently enriches colon cancer initiating cells. Ann Surg Oncol 15: 2927-2933, 2008.

14. Pilati P, Mocellin S, Bertazza L, et al: Prognostic value of putative circulating cancer stem cells in patients undergoing hepatic resection for colorectal liver metastasis. Ann Surg Oncol 19: 402-408, 2012

15. Screaton GR, Bell MV, Jackson DG, Cornelis FB, Gerth U and Bell JI: Genomic structure of DNA encoding the lymphocyte homing receptor $\mathrm{CD} 44$ reveals at least 12 alternatively spliced exons. Proc Natl Acad Sci USA 89: 12160-12164, 1992.

16. Screaton GR, Bell MV, Bell JI and Jackson DG: The identification of a new alternative exon with highly restricted tissue expression in transcripts encoding the mouse Pgp-1 (CD44) homing receptor. Comparison of all 10 variable exons between mouse, human, and rat. J Biol Chem 268: 12235-12238, 1993.

17. Rall CJ and Rustgi AK: CD44 isoform expression in primary and metastatic pancreatic adenocarcinoma. Cancer Res 55 $1831-1835,1995$.

18. Bhatavdekar JM, Patel DD, Chikhlikar PR, et al: Overexpression of CD44: a useful independent predictor of prognosis in patients with colorectal carcinomas. Ann Surg Oncol 5: 495-501, 1998.

19. Seiter S, Arch R, Reber S, et al: Prevention of tumor metastasis formation by anti-variant CD44. J Exp Med 177: 443-455, 1993.

20. Gunthert U, Hofmann M, Rudy W, et al: A new variant of glycoprotein CD44 confers metastatic potential to rat carcinoma cells. Cell 65: 13-24, 1991

21. Tremmel M, Matzke A, Albrecht I, et al: A CD44v6 peptide reveals a role of CD44 in VEGFR-2 signaling and angiogenesis Blood 114: 5236-5244, 2009

22. Alves CS, Yakovlev S, Medved L and Konstantopoulos K: Biomolecular characterization of CD44-fibrin(ogen) binding: distinct molecular requirements mediate binding of standard and variant isoforms of CD44 to immobilized fibrin(ogen). J Biol Chem 284: 1177-1189, 2009.

23. Alves CS, Burdick MM, Thomas SN, Pawar $P$ and Konstantopoulos K: The dual role of CD44 as a functional $\mathrm{P}$-selectin ligand and fibrin receptor in colon carcinoma cell adhesion. Am J Physiol Cell Physiol 294: C907-C916, 2008.

24. Hanley WD, Napier SL, Burdick MM, Schnaar RL, Sackstein R and Konstantopoulos K: Variant isoforms of CD44 are P- and $\mathrm{L}$-selectin ligands on colon carcinoma cells. FASEB J 20 337-339, 2006.

25. Nemerson Y: Tissue factor and hemostasis. Blood 71: 1-8, 1988.

26. Carson SD and Brozna JP: The role of tissue factor in the production of thrombin. Blood Coagul Fibrinolysis 4: 281-292, 1993.

27. Milsom CC, Yu JL, Mackman N, et al: Tissue factor regulation by epidermal growth factor receptor and epithelial-to-mesenchymal transitions: effect on tumor initiation and angiogenesis. Cancer Res 68: 10068-10076, 2008.

28. Milsom C, Magnus N, Meehan B, Al-Nedawi K, Garnier D and Rak J: Tissue factor and cancer stem cells: is there a linkage? Arterioscler Thromb Vasc Biol 29: 2005-2014, 2009.

29. Milsom C, Yu J, May L, et al: The role of tumor-and host-related tissue factor pools in oncogene-driven tumor progression. Thromb Res 120 (Suppl 2): S82-S91, 2007.
30. Milsom C, Anderson GM, Weitz JI and Rak J: Elevated tissue factor procoagulant activity in CD133-positive cancer cells. J Thromb Haemost 5: 2550-2552, 2007.

31. Seto S, Onodera H, Kaido T, et al: Tissue factor expression in human colorectal carcinoma: correlation with hepatic metastasis and impact on prognosis. Cancer 88: 295-301, 2000.

32. Nitori N, Ino Y, Nakanishi Y, et al: Prognostic significance of tissue factor in pancreatic ductal adenocarcinoma. Clin Cancer Res 11: 2531-2539, 2005.

33. Oliveira LR, Castilho-Fernandes A, Oliveira-Costa JP, Soares FA, Zucoloto S and Ribeiro-Silva A: CD $44^{+} / \mathrm{CD} 133^{+}$ immunophenotype and matrix metalloproteinase-9 influences on prognosis of early stage oral squamous cell carcinoma patients. Head Neck: Nov 1, 2013 (Epub ahead of print).

34. Rentala S, Chintala R, Guda M, Chintala M, Komarraju AL and Mangamoori LN: Atorvastatin inhibited Rho-associated kinase 1 (ROCK1) and focal adhesion kinase (FAK) mediated adhesion and differentiation of CD133CD44 prostate cancer stem cells. Biochem Biophys Res Commun 441: 586-592 2013.

35. Liu Q, Li JG, Zheng XY, Jin F and Dong HT: Expression of CD133, PAX2, ESA, and GPR30 in invasive ductal breast carcinomas. Chin Med J 122: 2763-2769, 2009.

36. Lee SM, Lee KE, Chang HJ, et al: Prognostic significance of CD44s expression in biliary tract cancers. Ann Surg Oncol 15: 1155-1160, 2008

37. Gansauge F, Gansauge S, Zobywalski A, et al: Differential expression of CD44 splice variants in human pancreatic adenocarcinoma and in normal pancreas. Cancer Res 55: 5499-5503, 1995.

38. Saigusa S, Tanaka K, Toiyama Y, et al: Correlation of CD133, OCT4, and SOX2 in rectal cancer and their association with distant recurrence after chemoradiotherapy. Ann Surg Oncol 16: 3488-3498, 2009

39. Miki J, Furusato B, Li H, et al: Identification of putative stem cell markers, CD133 and CXCR4, in hTERT-immortalized primary nonmalignant and malignant tumor-derived human prostate epithelial cell lines and in prostate cancer specimens. Cancer Res 67: 3153-3161, 2007.

40. Hermann PC, Huber SL, Herrler T, et al: Distinct populations of cancer stem cells determine tumor growth and metastatic activity in human pancreatic cancer. Cell Stem Cell 1: 313-323, 2007.

41. Sun Y,Han J, Lu Y, Yang X and Fan M: Biological characteristics of a cell subpopulation in tongue squamous cell carcinoma. Oral Dis 18: 169-177, 2012.

42. Liu Y, Jiang P, Capkova K, et al: Tissue factor-activated coagulation cascade in the tumor microenvironment is critical for tumor progression and an effective target for therapy. Cancer Res 71: 6492-6502, 2011

43. Rong Y, Post DE, Pieper RO, Durden DL, Van Meir EG and Brat DJ: PTEN and hypoxia regulate tissue factor expression and plasma coagulation by glioblastoma. Cancer Res 65: 1406-1413, 2005.

44. Fernandez PM and Rickles FR: Tissue factor and angiogenesis in cancer. Curr Opin Hematol 9: 401-406, 2002. 